\title{
High-Energy Atmospheric Reentry Test Aerothermodynamic Analysis
}

\author{
Alireza Mazaheri* \\ NASA Langley Research Center, Hampton, Virginia 23681
}

DOI: $10.2514 / 1 . \mathrm{A} 32407$

\begin{abstract}
The aerothermodynamic environment around an $8.3 \mathrm{~m}$ High-Energy Atmospheric Reentry Test vehicle is assessed. Twelve nose-shape configurations are generated, and their responses at the peak heating trajectory point are compared against the baseline nose shape. The possibility of a two-piece thermal protection system design at the nose is also considered, as are the surface catalytic affects of the aeroheating environment of such a configuration. Based on these analyses, an optimum nose shape is proposed to minimize the surface heating. The heat flux sensitivity of the optimum geometry to the angle of attack variations are also studied. A recommendation is also made for a two-piece thermal protection system design, for which the surface catalytic uncertainty associated with the jump in heating at the nose-inflatable aerodynamic decelerator juncture is reduced by a minimum of $93 \%$. In this paper, the aeroshell is assumed to be rigid, and the inflatable fluid interaction effect is left for future investigations.
\end{abstract}

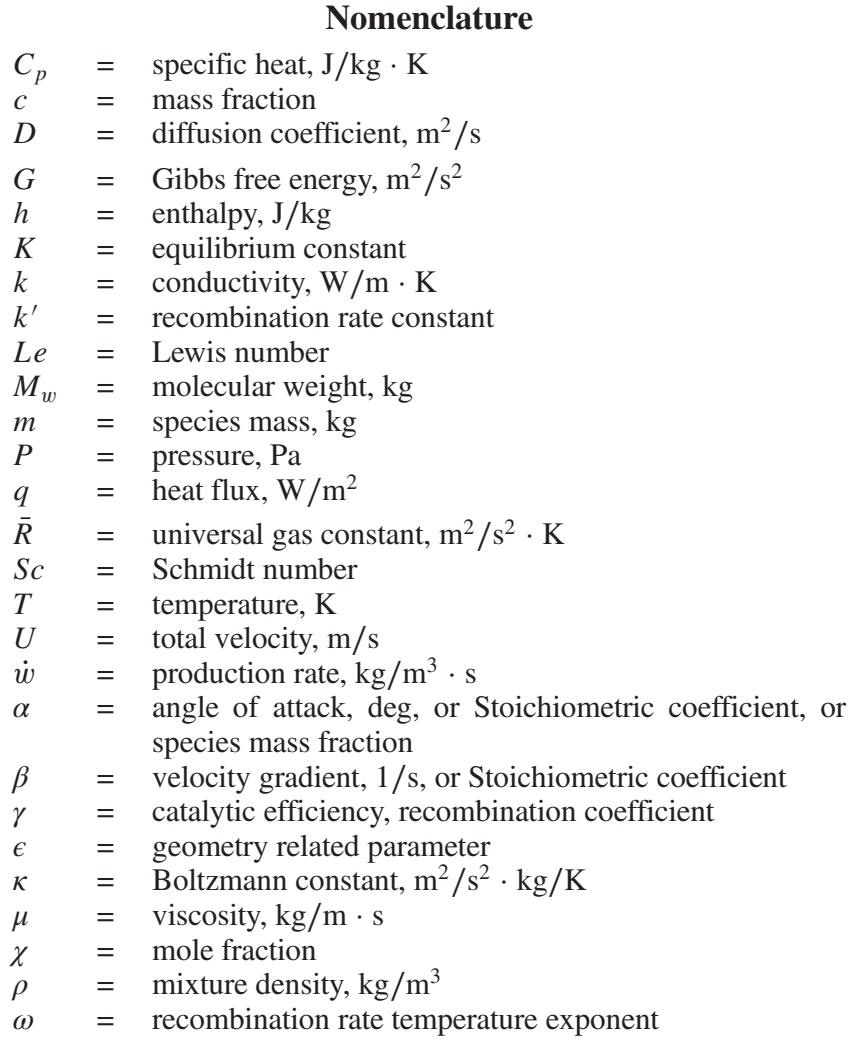

Subscripts

$\begin{array}{ll}e & =\text { edge } \\ \text { diff } & =\text { diffusion or catalytic } \\ w & =\text { wall } \\ s & =\text { species } s \\ \text { stg } & =\text { stagnation point }\end{array}$

Received 27 April 2012; revision received 5 July 2012; accepted for publication 19 July 2012; published online 1 March 2013. This material is declared a work of the U.S. Government and is not subject to copyright protection in the United States. Copies of this paper may be made for personal or internal use, on condition that the copier pay the $\$ 10.00$ per-copy fee to the Copyright Clearance Center, Inc., 222 Rosewood Drive, Danvers, MA 01923 include the code 1533-6794/13 and \$10.00 in correspondence with the CCC.

*Research Scientist, Aerothermodynamics Branch, M/S 408A; Ali.R .Mazaheri@nasa.gov. Senior Member AIAA.

\section{Introduction}

$\mathbf{H}$ YPERSONIC inflatable aerodynamic decelerators (HIAD) [1] are being developed for next generation atmospheric entry vehicles. Inflatables offer increased payload volume in a fraction of the launch vehicle shroud and have the potential to deliver more payload mass to the surface for a given set of trajectory constraints compared with rigid aeroshells. Another advantage is that the inflatable aerodynamic decelerators (IAD) [2] also allow access to the payload after the vehicle is integrated for launch.

IADs are still in the developmental phase with technical challenges still to be resolved. These challenges include the aerothermodynamic environment and the associated thermal protection systems (TPS) response of the IAD during atmospheric entry. The Inflatable Reentry Vehicle Experiment (IRVE)-III is addressing one of the steps on the development path of the HIAD, which is a more operationally realistic flight environment than its predecessor IRVE-II [3]. The High-Energy Atmospheric Reentry Test (HEART), which will build on the IRVE flights and developments, focuses on understanding the scale effects of an IAD. HEART is proposed to be an $8.3 \mathrm{~m}$ HIAD entering the Earth ballistically from the International Space Station (ISS) as a means to bring back unnecessary or excess payload (e.g., space junk or garbage) from the ISS.

For this study, baseline HEART geometry was analyzed and optimized for its aeroheating environment during the Earth entry. For the shape optimization, the author considered several nose-IAD shapes and proposes an optimum configuration. The effects of angle of attack on the surface aeroheating are also analyzed. Ranges of TPS surface catalytic coefficients are considered, and a recommendation is made to mitigate the catalytic uncertainty. Note that in this paper, the aeroshell is assumed to be rigid, and the inflatable fluid interaction effect that becomes important mainly in the low supersonic part of the trajectory is left for future investigations.

\section{Baseline Configuration}

This section discusses an aeroheating analysis conducted on the HEART baseline geometry, which consisted of an elliptical nose attached to an $8.3 \mathrm{~m}$ diameter IAD with a $55 \mathrm{deg}$ half-angle cone. The baseline configuration was provided by the HEART packaging team from their initial study without a complete aeroheating analysis. Figure 1 schematically shows the HEART baseline geometry. The rigid nose piece, which is elliptical for packaging purposes, creates a juncture at the first IAD torus, known as T1. In this baseline configuration, a TPS material wraps around the outer surfaces of the $\mathrm{IAD}$ and the nose entirely.

This paper presents an aerothermodynamic environment analysis on this baseline geometry to identify the peak heating point along the trajectory. The trajectory that was used in these simulations is plotted 


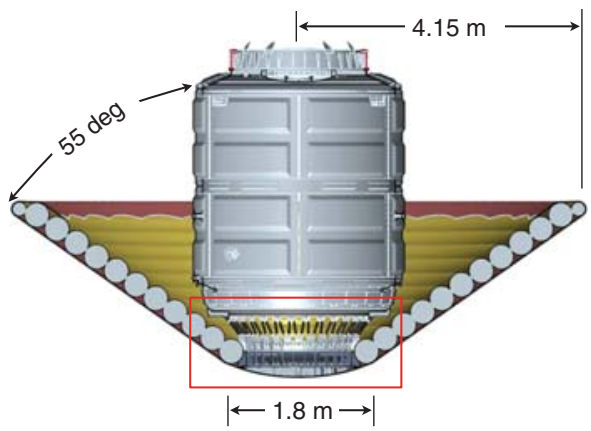

a)

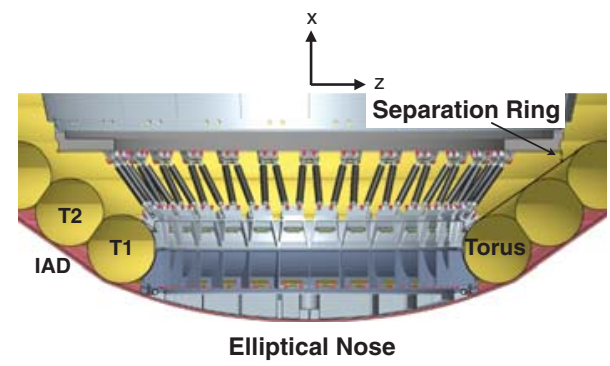

b)

Fig. 1 Baseline geometry for a) $8.3 \mathrm{~m}$ HEART geometry with a 55 deg half-angle cone, and b) close up around the nose.

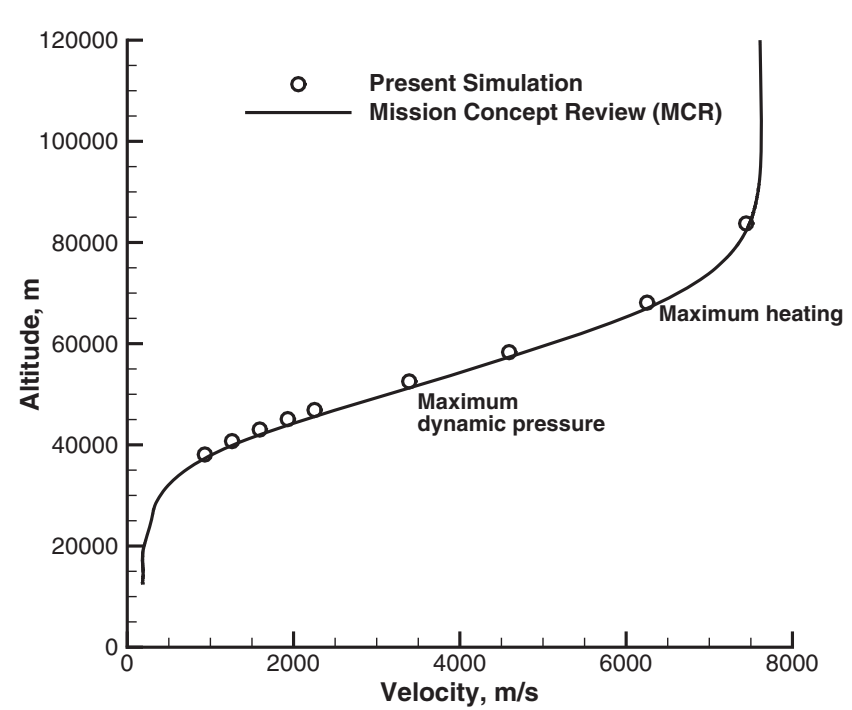

Fig. 2 Trajectory points used in the present simulation.

in Fig. 2. Table 1 shows the tabulations of the freestream conditions for the selected points shown as open symbols in Fig. 2. This study assumed laminar five-species air with a thermal equilibrium condition. Surface temperature was computed using a radiative equilibrium boundary condition with an assumed constant emissivity of 0.85 . The surface catalytic condition was assumed to be fully catalytic (FC), in which all atoms were allowed to recombine on the surface. This condition provided the upper bound for the surface heat flux. Unless otherwise stated, the computations were performed using the LAURA-5 [4] code at a constant angle of attack of 10 deg. LAURA has been used on other vehicles, such as Fire II [5], the Space Shuttle Orbiter [6,7], and X-37, and is also being used for Mars Science Laboratory (MSL) [8] and Multi-Purpose Crew Vehicle (MPCV) missions [9].

Figure 3 shows the centerline surface heat flux for the baseline geometry at the trajectory points T2163-T2292. These solutions were performed on two separate 3-D structured grids, both with the same number of surface points but with a factor of two difference in the number of points normal to the body. Doubling the number of points did not change the surface heat flux values more than $5 \%$, and therefore, the solutions were confirmed to be grid-converged (Fig. 4).

The results in Fig. 3 showed that the surface heating increases from point T2163 to T2255 before it decreased at point T2292. Point T2255, which was a Mach 21 point on the entry trajectory, was therefore the peak heating point for this trajectory. This trajectory does not have a dual-peak pulse, and thus, no further analysis is needed to locate the peak heating condition.

The centerline surface heating profile, however, presented a double peak heating, which was due to the rapid curvature change from the nose to the IAD section (see Fig. 1). Note that simulations were conducted at $\alpha=10 \mathrm{deg}$. The nose-IAD juncture closest to the stagnation point produced a significant rise in the surface heat flux. The maximum surface heat flux at this peak heating point T2255 was about $40 \mathrm{~W} / \mathrm{cm}^{2}$. The uneven distribution flattened out as the vehicle traveled down the trajectory, but its presence was not desirable, as this could constrain the vehicle design from further considerations. For example, because the IAD is not rigid, the TPS fabric would have some irregularity between the tori, which is known as TPS scalloping. The TPS scalloping itself could cause some irregularity to the surface heat flux. However, if the smooth surface heat distribution were uneven, the TPS scalloping could augment the surface heating. Thus, it is desirable to have a geometry that produces a relatively smooth surface heating profile but still fulfills packaging constraints.

The surface catalytic contribution to convective heat flux is calculated by comparing the fully catalytic surface heat flux with one computed with a noncatalytic (NC) surface condition. The noncatalytic surface is defined as a surface that does not allow species recombinations at the surface. A noncatalytic surface eliminates the catalytic heating portion of the heat flux and results in a much lower heat flux than that produced by a fully catalytic surface. This effect is illustrated in Fig. 5, which shows a factor of 3 in catalytic uncertainty. The difference in heat flux due to catalysis was highest at the peak heating and decreased rapidly at lower altitudes. For example, the catalytic uncertainty factor decreased from about 3 to about 2 at the next trajectory point after the peak heating point (Fig. 6). In Fig. 6, a code-to-code comparison was made and excellent agreement was achieved between the LAURA-5 [4] and the DPLR [10] codes. In this comparison, the grid was first adapted and aligned with the bow shock using the LAURA-5 code. The same adapted grid was then used for the DPLR runs with no additional grid alignment.

Figure 6 also shows a LAURA-5 supercatalytic solution. The supercatalytic condition was defined by replacing the species concentration at the wall with the freestream species compositions. The comparison showed that the fully catalytic and the supercatalytic results were almost identical. This means that the $100 \%$ catalytic efficiency at the wall resulted in the same species concentration as the freestream because of a relatively low surface temperature. The author observed similar results for the other trajectory points.

Table 1 Freestream conditions for the trajectory points shown in Fig. 2

\begin{tabular}{cccccc}
\hline \hline Time, $\mathrm{s}$ & Altitude, $\mathrm{km}$ & $\rho_{\infty}, \mathrm{kg} / \mathrm{m}^{3}$ & $U_{\infty}, \mathrm{m} / \mathrm{s}$ & $T, \mathrm{~K}$ & Mach \\
\hline 2163 & 84 & $8.912 \mathrm{E}-06$ & 7443.7 & 213.3 & 25 \\
2255 & 68 & $8.914 \mathrm{E}-05$ & 6252.0 & 224.6 & 21 \\
2292 & 58 & $3.383 \mathrm{E}-04$ & 4592.9 & 241.3 & 15 \\
2311 & 52 & $7.056 \mathrm{E}-04$ & 3391.2 & 253.4 & 11 \\
2328 & 47 & $1.458 \mathrm{E}-03$ & 2252.9 & 257.3 & 7 \\
2334 & 45 & $1.848 \mathrm{E}-03$ & 1930.2 & 256.9 & 6 \\
2340 & 43 & $2.471 \mathrm{E}-03$ & 1593.5 & 252.3 & 5 \\
2348 & 41 & $3.434 \mathrm{E}-03$ & 1260.1 & 247.0 & 4 \\
2356 & 38 & $5.087 \mathrm{E}-03$ & 932.9 & 240.3 & 3 \\
\hline \hline
\end{tabular}




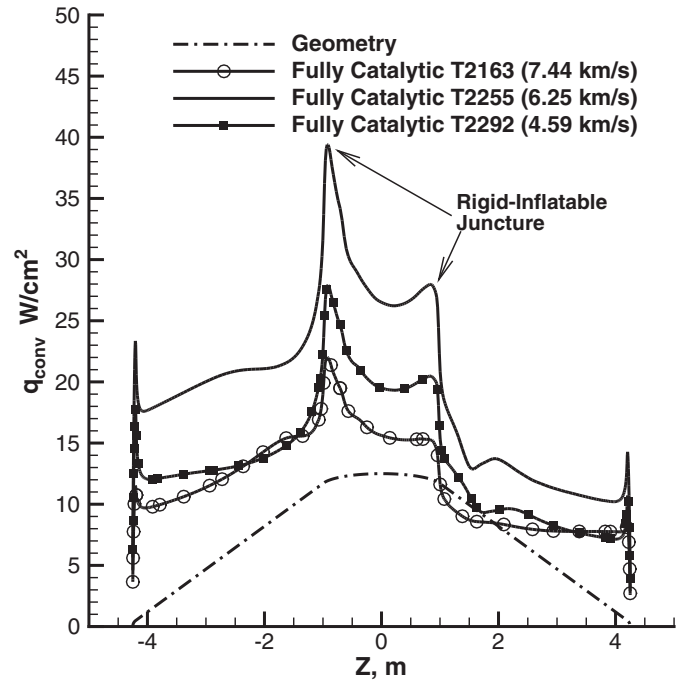

Fig. 3 Centerline surface heat flux on the baseline geometry.

The trajectory point T2311 is the maximum dynamic pressure point. The corresponding pressure contour plot is shown in Fig. 7. In a subset of Fig. 7, the adapted grid is shown. A wall cell Reynolds number of 0.1 was imposed during the adaptation process to ensure a more accurate viscous layer representation and surface heat flux computation. During the adaptation process the grid was refined across the shock discontinuity for a crisper shock location prediction and better numerical accuracy.

\section{Nose-Shape Optimization}

The aeroheating analysis of the baseline HEART geometry presented in the previous section showed an unevenness in the surface heat flux, more specifically a double peak heating at the noseIAD juncture. This section presents a series of nose optimizations to reduce the surface heat flux and achieve a relatively smoother surface heating profile. The optimization was done manually and sequentially. The nose shapes were modified through a series of tries and errors according to their surface heat flux responses. There were some geometrical constraints from packaging and manufacturing standpoints that were imposed before the optimization. At the beginning of the investigation, there was a constraint that the IAD should clear the separation ring at the shoulder, shown in Fig. 1. The baseline configuration, shown in Fig. 1, had the IAD clearing this

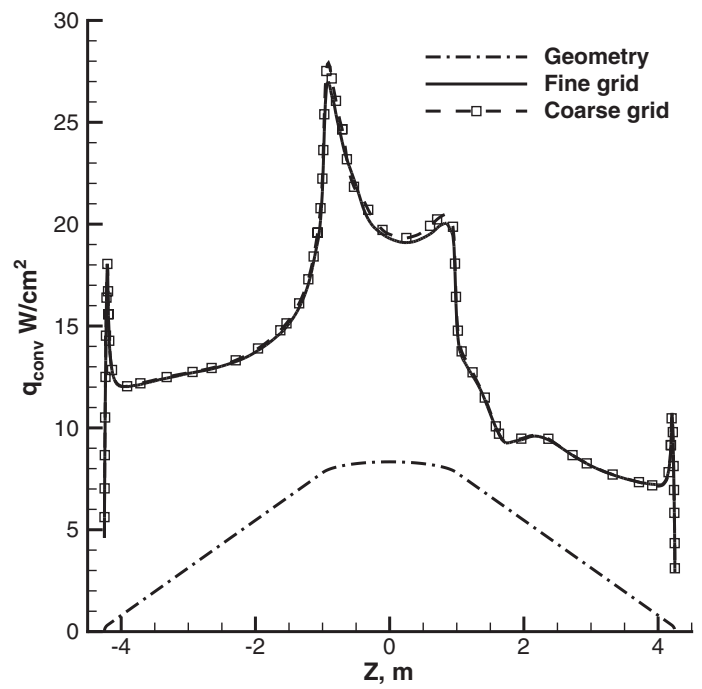

Fig. 4 Surface heat flux comparison at a trajectory point on two different grid resolutions with fully catalytic and radiative equilibrium surface boundary conditions.

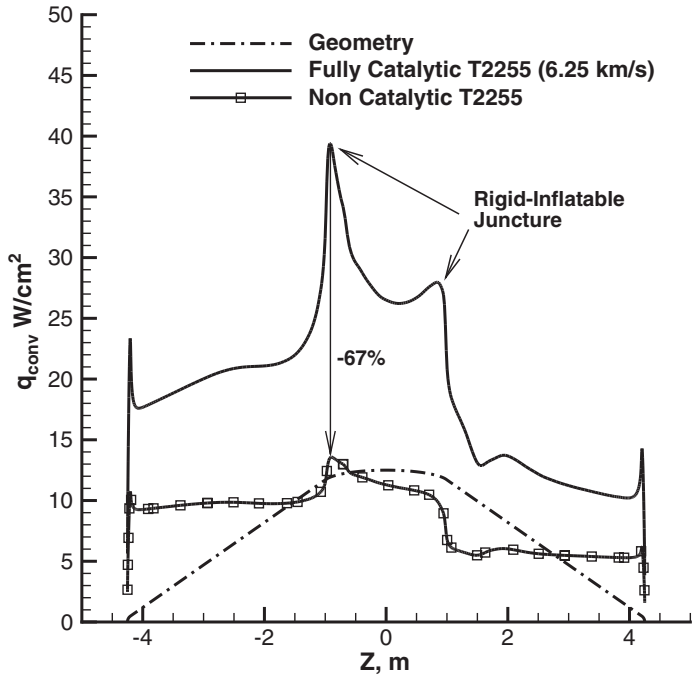

Fig. 5 Maximum surface catalytic uncertainty for the surface heat flux on the baseline geometry.

shoulder by just over 2 in. However, it was expected that the IAD would deflect under its load such that the IAD would contact the shoulder. Therefore, it was suggested to move the IAD back to where it would touch the shoulder when it had no load. This provided an additional 2 in. in the direction of the minor diameter of the baseline nose.

During early optimization, the backside portion of the IAD section was allowed to curve. Initially this was not a constraint, but it was suggested that any curvature to the IAD section would complicate the fabrication process, and thus must be avoided. The overall vehicle diameter of $8.3 \mathrm{~m}$ and the half-angle cone of $55 \mathrm{deg}$ were also fixed to limit the parameters.

Figure 8 shows a series of the nose shapes conducted on the HEART baseline geometry. The dotted lines correspond to the baseline configuration, while the solid lines represent alternative geometries. Some of the modified geometries require slightly curved IADs, but not beyond the fourth torus T4, and some had the exact same IAD section as the baseline. Nose shapes that were elliptical did not have an analytical representation, as they were generated based on past experiences and examination of the surface heating profile of the baseline geometry.

Separate 3-D structured grids were generated for each of the Type 1-12 geometries. To do a better comparison with the baseline

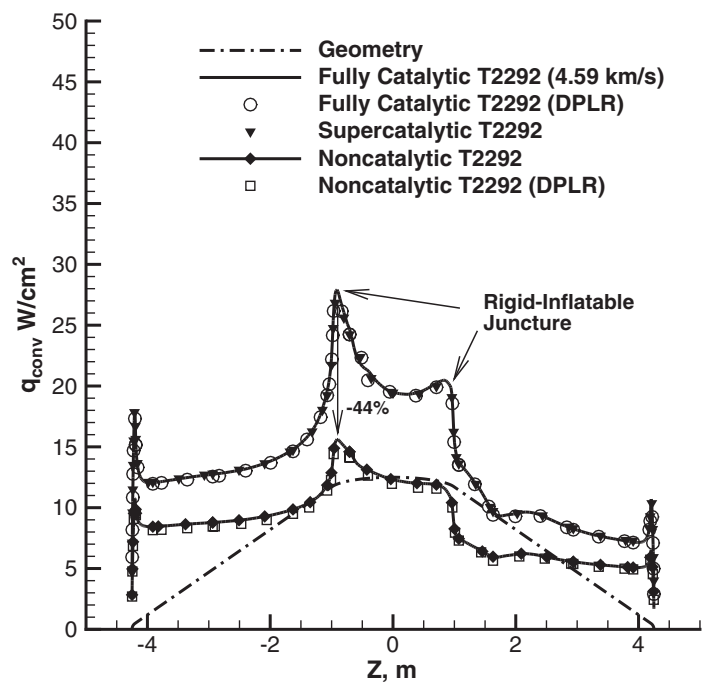

Fig. 6 Catalytic uncertainty and LAURA [4] — DPLR [5] code-to-code comparisons for the surface heat flux on the baseline geometry at T2292. 


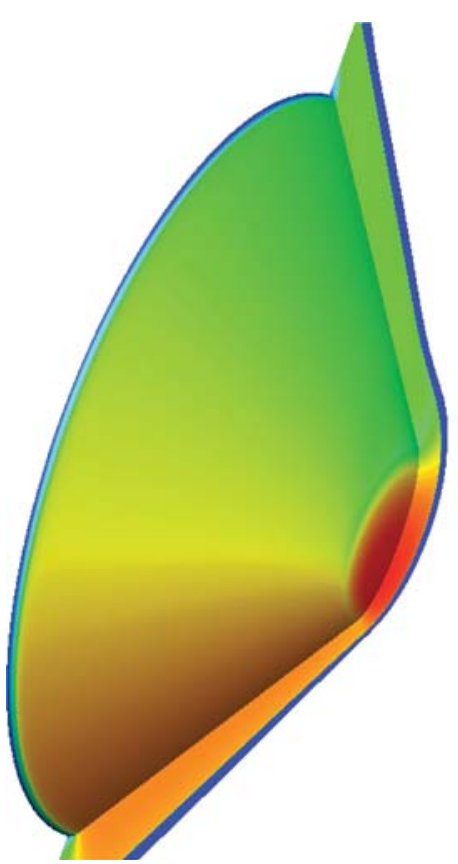

a)
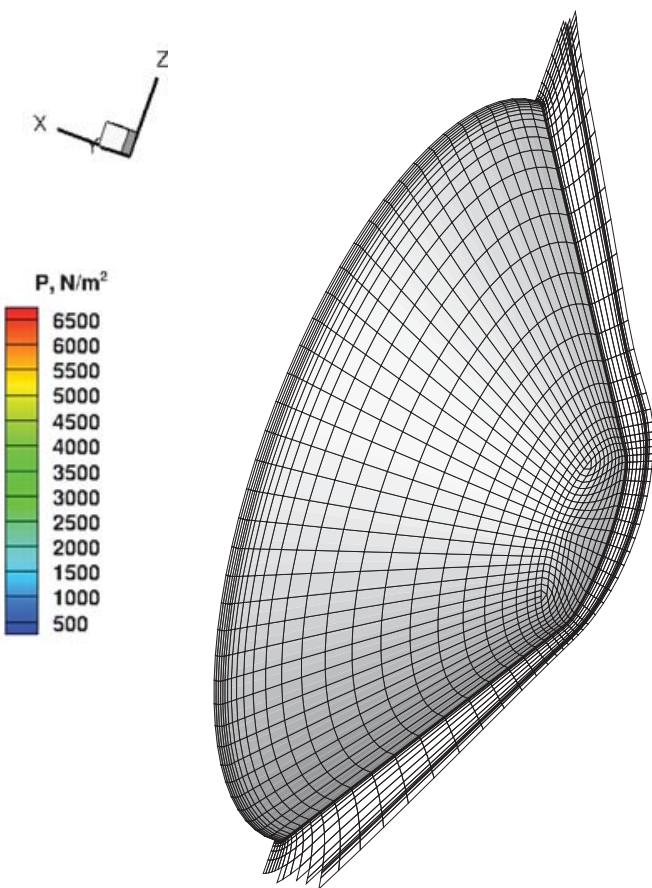

b)

Fig. 7 Schematics of a) pressure contour plot at the maximum pressure point on the baseline geometry, and b) structure grid used in the simulation (for clarity every fourth grid point is shown).

geometry, the surface grids were kept as close to the baseline grid as possible. The total number of volume cells was also kept identical. During the simulation, the grids were adapted, aligned, and clustered around the bow shock, similar to the one shown in Fig. $7 \mathrm{~b}$.
Only the peak heating point T2255 with a fully catalytic surface and radiative equilibrium boundary condition is discussed here. The geometry effects were based on the surface heating distribution and its maximum value as compared with the baseline geometry. Among
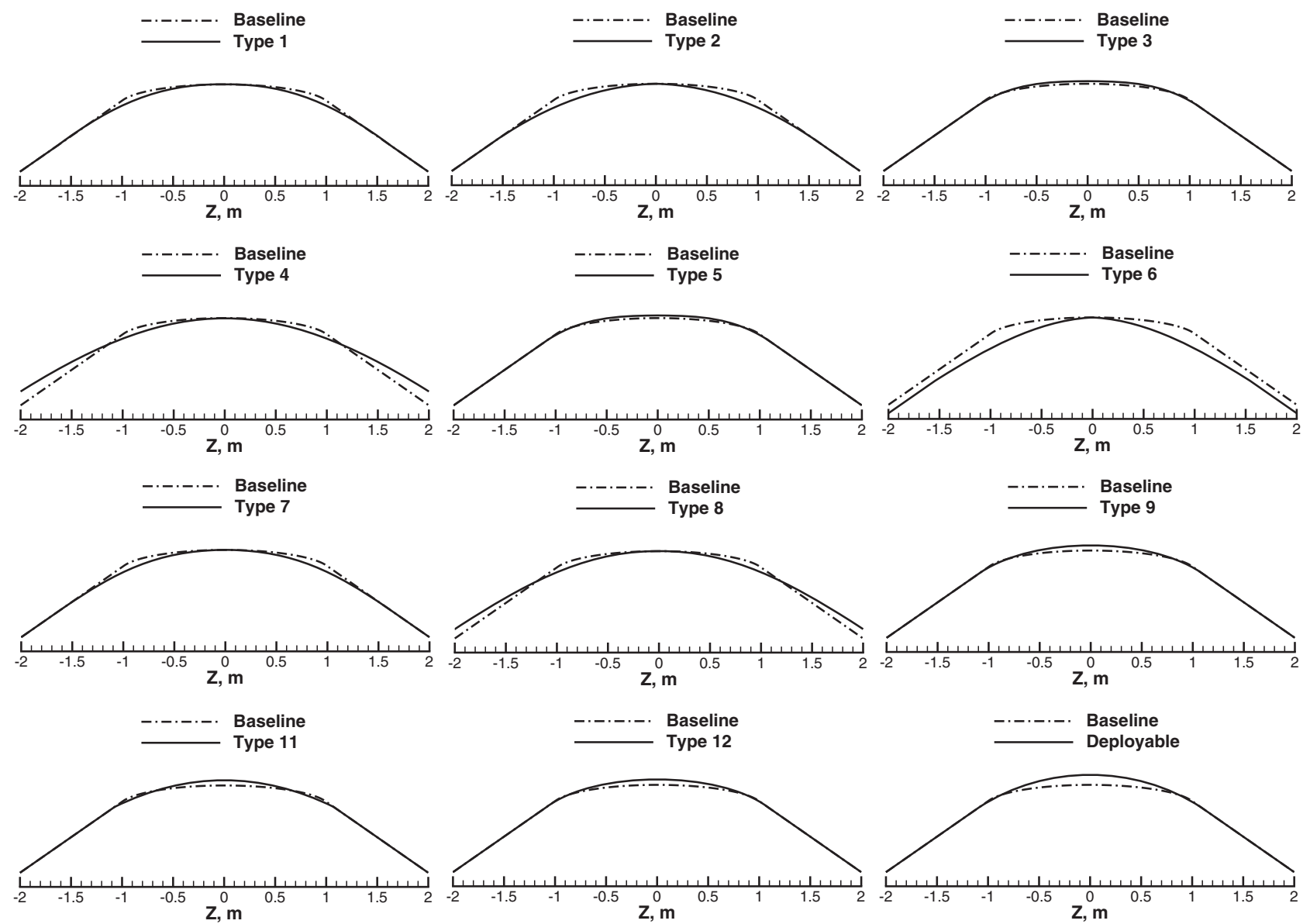

Fig. 8 Series of nose-shape optimizations for the $8.3 \mathrm{~m}$ HEART vehicle with 55-deg half-angle cone. 


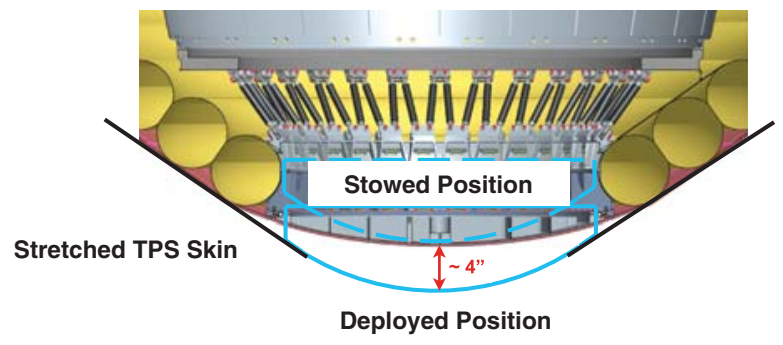

Fig. 9 Deployable concept for the HEART geometry.

the presented configurations, Types 5, 9, 11, 12, and the deployable nose concept did not require any changes to the IAD curvature. The remaining geometries added relatively small curvature to the IAD section up to the forth torus T4. Types 5, 9, and 12 were nonspherical semi-elliptical nose configurations. All others were spherical. Types 9 and 11 had a nose that was moved, respectively, 1 and 2 in. forward compared with the baseline geometry. Type 12 was similar to Type 11 except that the nose was no longer spherical. The center point of the Type 12 nose was about 2 in. forward relative to the baseline geometry.

One of the interesting configurations studied here is the deployable nose concept proposed by Hughes. ${ }^{\dagger}$ In this concept, illustrated schematically in Fig. 9, the nose is deployed from a stowed position at the entry point, which stretches the TPS fabric. This would provide a smaller nose radius, and therefore, the surface would be expected to experience a higher heat flux. However, the available unused room behind the deployed nose could be used as heat sink. The deployed nose, which is spherical, pushes the nose 4 in. forward with respect to the baseline grid. The deployable nose may impose some mechanical complexity that is beyond the scope of this paper, and therefore is not addressed here.

Figure 10 represents the centerline surface heat flux for all the geometries. A more detailed comparison is summarized and tabulated in Table 2. According to this table, all the modified geometries, except Type 6 , provided an $11-26 \%$ reduction in surface heating compared with the baseline geometry. Type 6 produced a slightly higher surface heat flux than the baseline geometry. All the modified geometries also experienced a $4-6 \%$ decrease in surface temperature.

The results summarized in Table 2 show that Types 4 and 8 provided the best surface heat flux responses. However, both Types 4 and 8 added curvatures to the IAD section up to T2 and were the choice amongst the curved IAD configurations. Types 11 and 12, on the other hand, bounded all the specified constraints and were the preferred choices within the straight IAD configurations. Type 12 provided a relatively smoother surface heating flux distribution, and therefore, was selected as the optimum geometry. The nose shape of the Type 12 geometry between $z= \pm 1 \mathrm{~m}$ can be expressed by a fourth-order polynomial as $x=-0.0514-0.000143 z+0.147 z^{2}$ $-0.000138 z^{3}+0.0684 z^{4}$.

\section{Angle-of-Attack Effects}

The HEART trajectory is ballistic with angle-of-attack variations of about \pm 5 deg. To account for the worst case, the optimizations were performed at an angle of attack (in this case $\alpha=10 \mathrm{deg}$ ) at which the vehicle experienced a maximum surface heating. In this section, the sensitivity of heating to the variations in the angle of attack is studied.

In the previous section, the shape optimization revealed that the Type 12, which did not require modification to the IAD section of the baseline geometry, experiences the lowest maximum surface heating among the fixed IAD geometries studied. For this reason, only this type is presented and analyzed in this section. Figure 11 shows the results of the angle-of-attack effect on surface heating. Open symbols

${ }^{\dagger}$ Private communication Hughes, S.J., HIAD Chief Engineer, NASA Langley Research Center; Stephen.J.Hughes@ @asa.gov. December 2011. in Fig. 11 indicate the solutions at $\alpha=10 \mathrm{deg}$ with a fully catalytic surface. The solid lines indicate solutions at $\alpha=0 \mathrm{deg}$. The results showed a relatively minor heat flux sensitivity to the entry angle, meaning that additional aeroheating analysis might not be needed to account for the angle-of-attack variations during the entry. This was because the Type 12 provided a relatively uniform nose curvature within \pm 10 deg relative to the freestream velocity. Thus, the angleof-attack effects on the surface heating prediction were minor. Note that the maximum surface heating of $34 \mathrm{~W} / \mathrm{cm}^{2}$ had been reduced by about $10 \%$ with reduction of $\alpha$. Similar results were seen for surface temperature. The IAD surface also experienced slightly lower surface heat flux and temperature at $\alpha=0 \mathrm{deg}$.

\section{Catalytic Surface Effect}

The HEART inner mold line (IML) must be protected with TPS material to survive the entry environment. The fabric TPS catalytic characteristics that will cover the IAD surface are yet to be determined. In this section, an assessment is made for a two-piece nose-IAD configuration using the Type 12 (optimum) geometry, where the nose was made of a separate nonablative TPS material. The IAD was still wrapped with a TPS fabric. Thus, the nose probably would have a surface catalytic property that is not necessarily similar to the IAD TPS. This configuration was, therefore, subject to an aerothermodynamic phenomenon known as catalytic jump.

Maximum catalytic uncertainty at the peak heating condition defined as the difference in fully and noncatalytic heating rates was about $250 \%$ for the Type 12 geometry with a fully wrapped TPS fabric (Fig. 12). The uncertainty translated to about $20 \mathrm{~W} / \mathrm{cm}^{2}$ variations in surface heating from a noncatalytic to a fully catalytic surface condition. Note that at this entry condition, the fully catalytic and supercatalytic conditions were predicting similar surface heating as presented previously in Fig. 6 .

For the two-piece nose-IAD catalytic jump analysis, the nose was assumed to be noncatalytic. This provided the worst catalytic jump condition, as all the atomic species present above the nose surface would be available for recombination on the IAD surface. The availability of free atoms above a catalytic surface caused a rapid jump in surface heating as a result of additional recombination energy.

The IAD surface catalysis was varied from a low-catalytic to a fully catalytic condition. Among these different surface catalyses, a surface with a variable catalytic property (such as reaction cured glass [RCG], which was used on the Space Shuttle Orbiter tiles) was also considered. Figure 13 shows the variation of RCG-catalytic efficiency with the temperature.

Surface catalytic efficiency $\gamma$, whether it was constant or variable, was used in the computation of the wall catalytic species recombination rate constant, defined as $[11,12]$

$$
k_{w s}=\frac{2 \gamma_{s}}{2-\gamma_{s}} \sqrt{\frac{\kappa T_{w}}{2 \pi m_{s}}}
$$

The computations were conducted at the peak heating condition and $\alpha=0$ deg. Figure 14 shows plots of surface heat flux and temperature along the centerbody of the geometry. The solid lines represent solutions with a uniform fully catalytic nose-IAD surface. Solutions with a uniform noncatalytic nose-IAD surface are shown with closed squares. Open circles with solid lines are the solutions with noncatalytic nose and partially catalytic IAD. The surface heat flux is presented in the plots in the left column. The plots shown in the right column are for the surface temperature.

The results showed that a $1 \%$ catalytic jump $(\gamma=0.01)$ leads to about a $170 \%$ increase in the surface heat flux at the nose-IAD juncture above the NC value. The heat flux then recovered to about $76 \%$ above the noncatalytic surface results on the IAD section, which corresponded to about $15 \mathrm{~W} / \mathrm{cm}^{2}$. The surface temperature jump was not as severe: $27 \%$ at the nose-IAD juncture and $14 \%$ on the IAD section. 

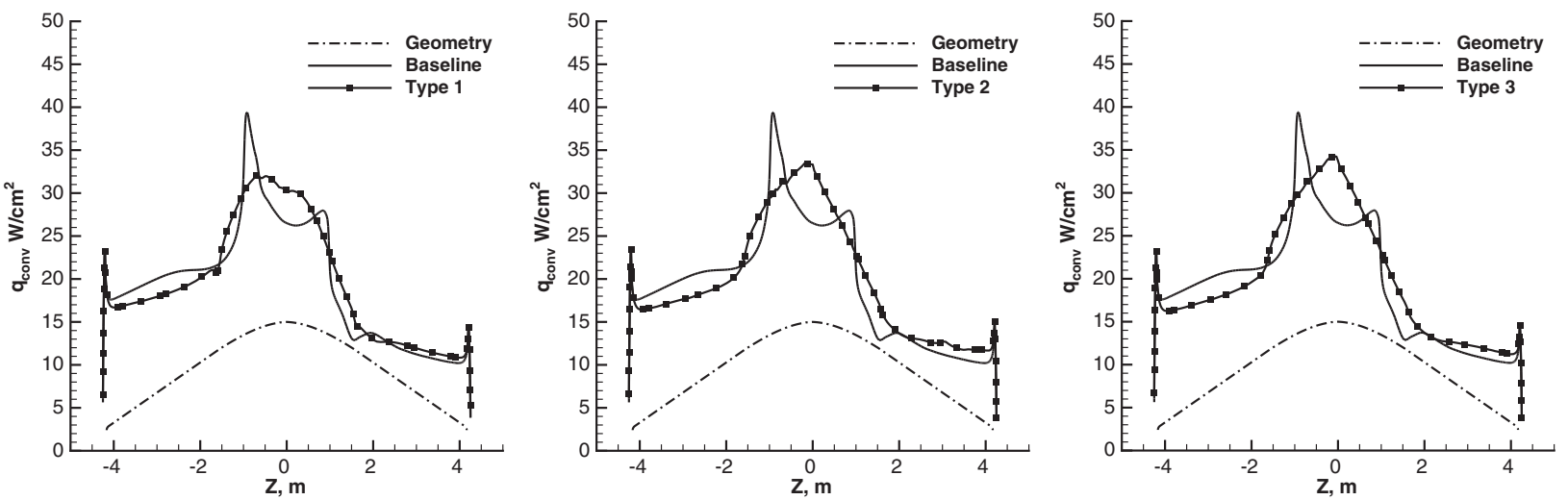

a) Type 1

b) Type 2

c) Type 3
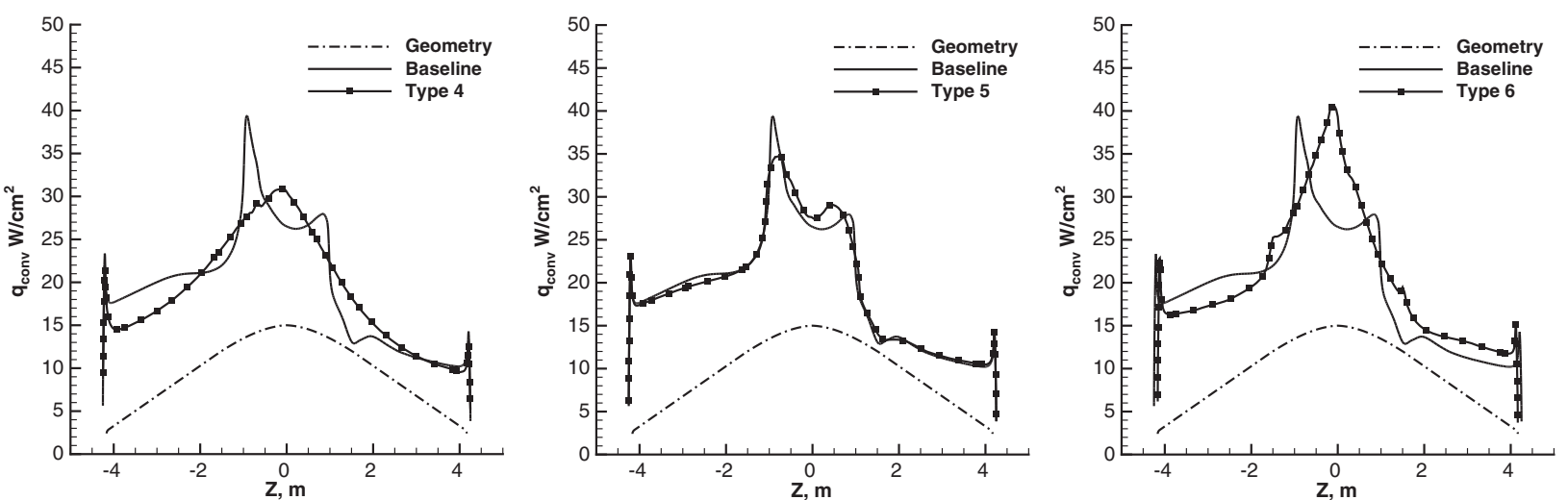

d) Type 4

e) Type 5

f) Type 6
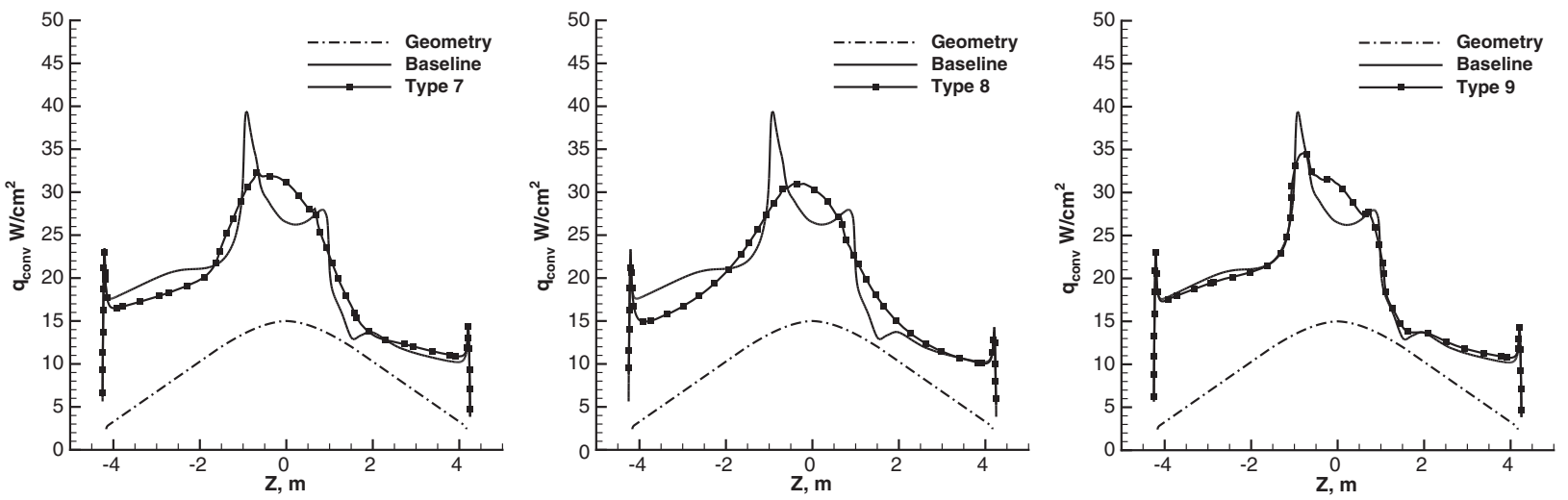

g) Type 7

h) Type 8

i) Type 9
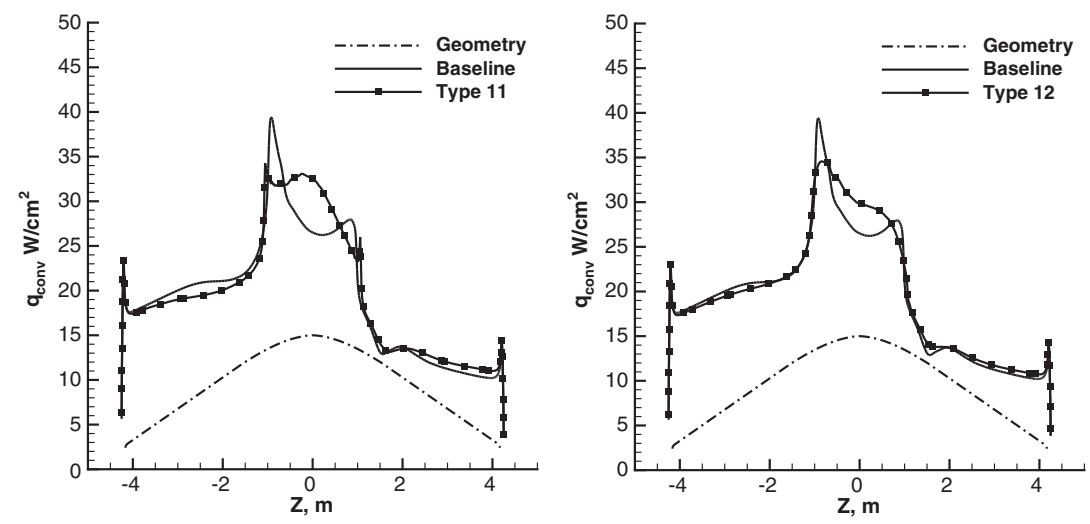

k) Type 12

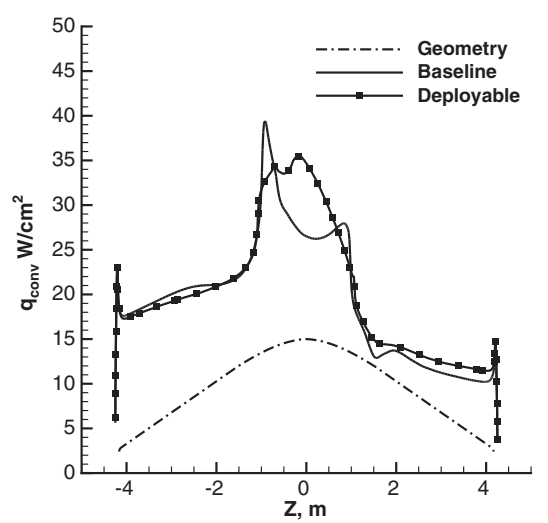

l) Deployable

Fig. 10 Centerline heating distribution for the nose-shape configurations presented in the Fig. 7. Note: $\alpha=10$ deg (concluded).

Results for an IAD with a RCG-catalytic surface predicted a $161 \%$ jump in the surface heat flux and a $23 \%$ jump in the surface temperature. These were slightly higher than the values predicted for the $1 \%$ catalytic jump. However, both the surface heat flux and the temperature values recovered to about 42 and $8 \%$ of the noncatalytic IAD results, respectively. The results with IAD surface catalytic 
Table 2 Maximum surface heat flux and percent reduction compared with the baseline nose; $\alpha=10 \mathrm{deg}$

\begin{tabular}{ccccccc}
\hline \hline Type & $\begin{array}{c}q_{\max }, \\
\mathrm{W} / \mathrm{cm}^{2}\end{array}$ & $\begin{array}{c}T_{\max }, \\
\mathrm{K}\end{array}$ & $1-\frac{q_{\text {baseline }}}{q}$ & $1-\frac{T_{\text {baseline }}}{T}$ & $\begin{array}{c}\text { Curved } \\
\text { IAD? }\end{array}$ & $\begin{array}{c}\text { Nose } \\
\text { Shape }\end{array}$ \\
\hline Baseline & 39 & 1690 & - & - & No & Elliptical \\
1 & 32 & 1600 & -0.22 & -0.06 & T1-T2 & Spherical \\
2 & 34 & 1630 & -0.15 & -0.04 & T1-T2 & Spherical \\
3 & 34 & 1630 & -0.15 & -0.04 & T1-T2 & Spherical \\
4 & 31 & 1600 & -0.26 & -0.06 & T1-T2 & Spherical \\
5 & 34 & 1630 & -0.15 & -0.04 & No & Elliptical \\
6 & 41 & 1710 & 0.05 & 0.01 & T1-T3 & Spherical \\
7 & 34 & 1630 & -0.15 & -0.04 & T1-T2 & Spherical \\
8 & 33 & 1620 & -0.18 & -0.04 & T1-T4 & Spherical \\
9 & 34 & 1630 & -0.15 & -0.04 & No & Spherical \\
11 & 33 & 1620 & -0.21 & -0.04 & No & Spherical \\
12 & 34 & 1630 & -0.15 & -0.04 & No & Elliptical \\
Deployable & 35 & 1650 & -0.11 & -0.04 & No & Spherical \\
\hline \hline
\end{tabular}

efficiencies of $0.03,0.05,0.1$, and 1.0 showed surface heat flux jumps of $252,316,397$, and $520 \%$ above the noncatalytic value, respectively. The surface temperature jumps were, respectively, 35 , 40,46 , and $54 \%$ higher than the values predicted with a uniform noncatalytic surface. However, results for the IAD surface catalysis above $5 \%$ recovered to the almost fully catalytic value on the IAD section (see Appendix A for more discussion on this.) The results of surface catalytic efficiency of up to $3 \%$ were bound within the maximum catalytic uncertainly prediction, meaning they were not above the uniform fully catalytic nose-IAD surface. Surface catalysis higher than $\gamma=0.03$ produced a jump that was significantly higher than the stagnation point for the fully catalytic surface. The results are further summarized and tabulated in Table 3. In this table the maximum catalytic augmentation factor MCAF and the maximum temperature augmentation factor MTAF are defined relative to the maximum heating value on a noncatalytic surface:

$$
\mathrm{MCAF}=\frac{q}{\left(q_{\max }\right)_{\gamma=0}} \quad \mathrm{MTAF}=\frac{T}{\left(T_{\max }\right)_{\gamma=0}}
$$

This table indicates that a $1 \%$ catalytic condition on the IAD TPS fabric leads to a surface heat flux jump at the nose-IAD juncture in excess of twice the noncatalytic stagnation point value. The surface temperature, however, was less sensitive to the catalytic jump.

Sensitivity of the nose surface catalysis to the catalytic jump was also studied by repeating these analyses with a RCG-catalytic nose and several IAD catalytic conditions ranging from 1 to $100 \%$. A case with a uniform nose-IAD RCG-catalytic condition was also considered. Figure 15 shows the surface heat flux and temperature variations along the centerline of the HEART geometry.

Comparing the RCG-catalytic nose results with the one presented with a noncatalytic nose in Fig. 14 shows that the RCG-catalytic nose had much more moderate surface heat flux jumps than the

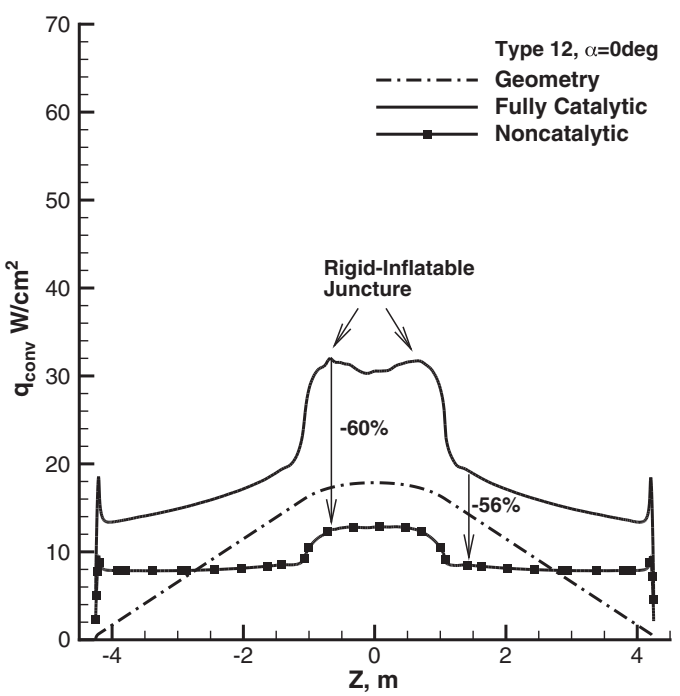

Fig. 12 Maximum catalytic uncertainty at the peak heating condition for the Type 12 geometry wrapped entirely with a fabric TPS.

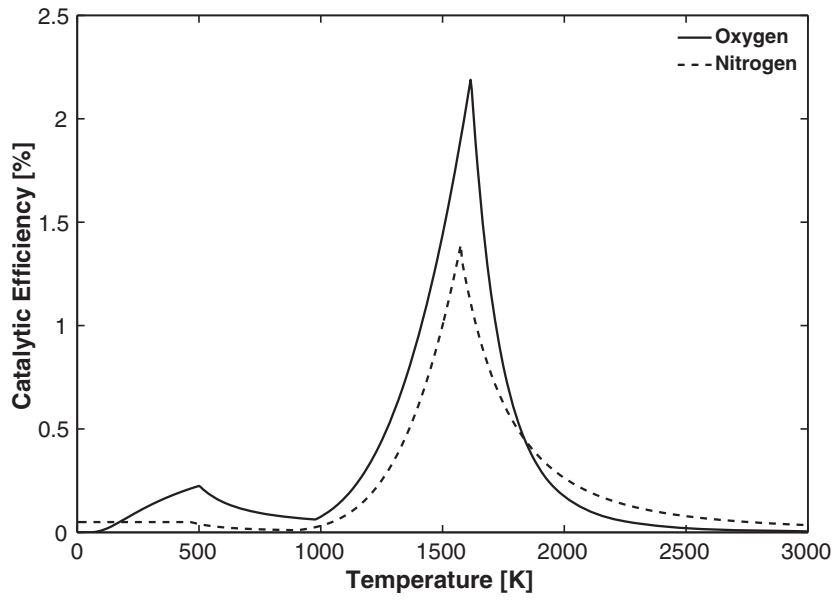

Fig. 13 RCG-Catalytic efficiency with temperature.

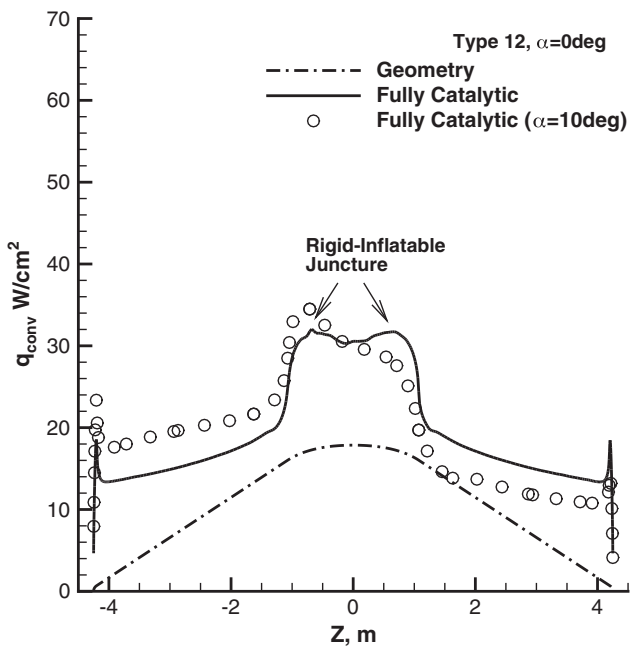

a) Surface heat flux distribution

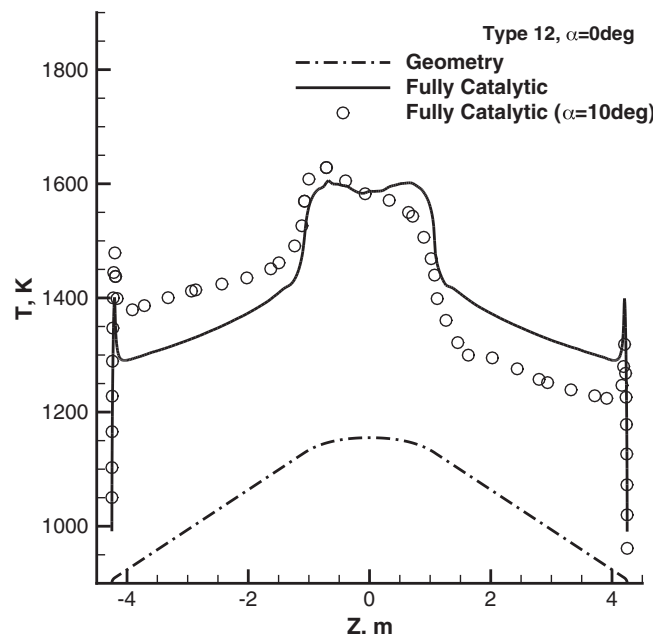

b) Surface temparature distribution

Fig. 11 Angle-of-attack effects on the Type 12 geometry. 


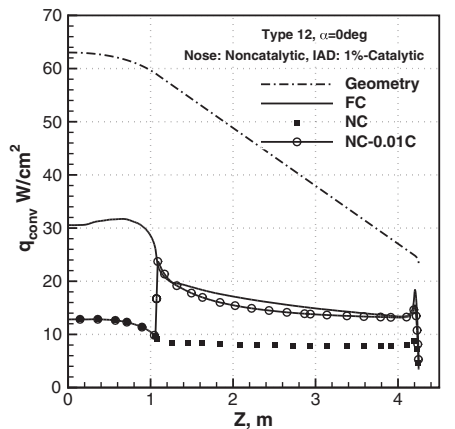

a) Surface heat flux distribution with $\gamma=1 \%$ catalysis on the IAD

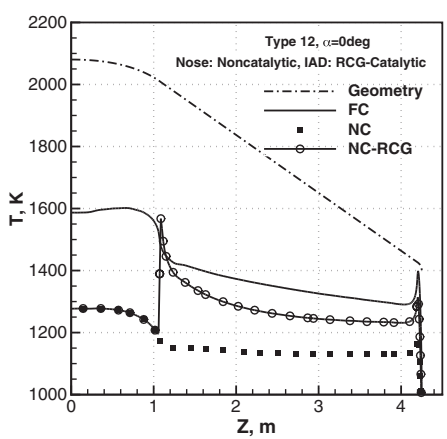

d) Surface temperature distribution with a RCG catalysis on the IAD

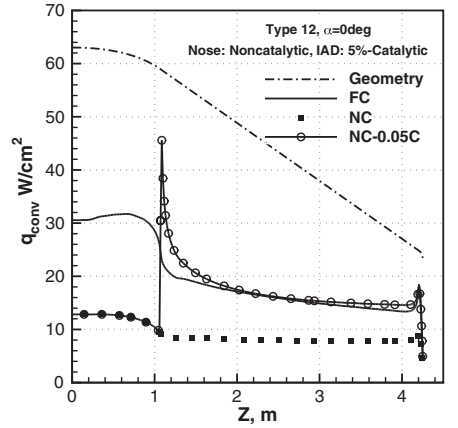

g) Surface heat flux distribution with a $5 \%$ catalytic IAD

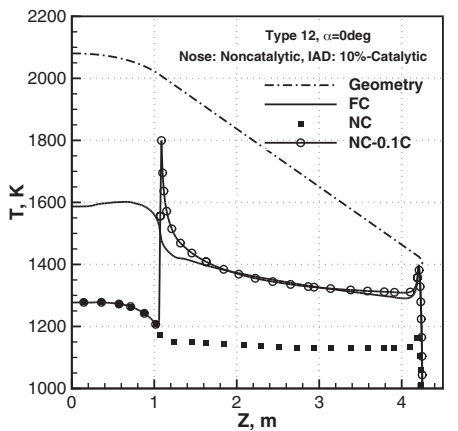

j) Surface temperature distribution with a $10 \%$ catalytic IAD

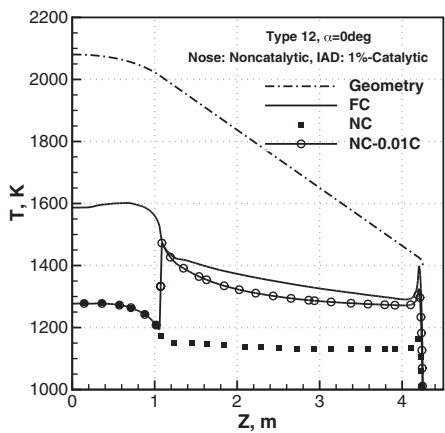

b) Surface temperature distribution with $\gamma=1 \%$ catalysis on the IAD

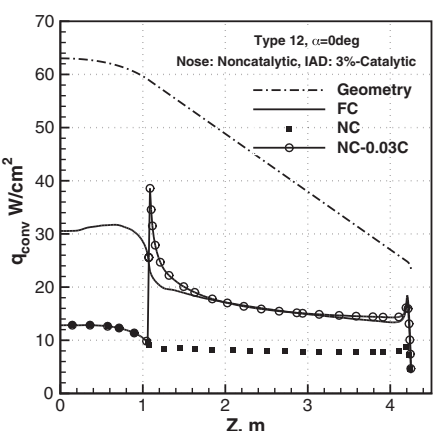

e) Surface heat flux distribution with $\gamma=3 \%$ catalysis on the IAD

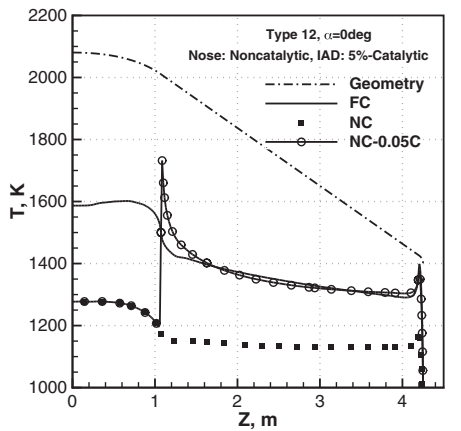

h) Surface temperature distribution with a $\mathbf{5 \%}$ catalytic IAD

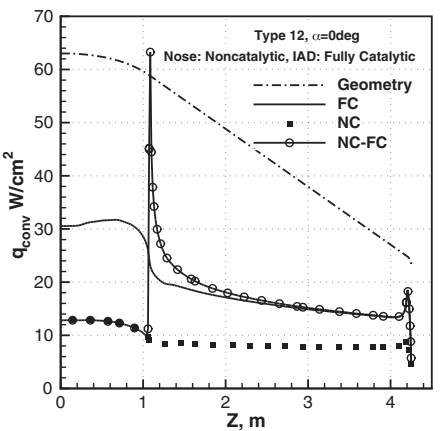

k) Surface heat flux distribution with a fully-catalytic IAD

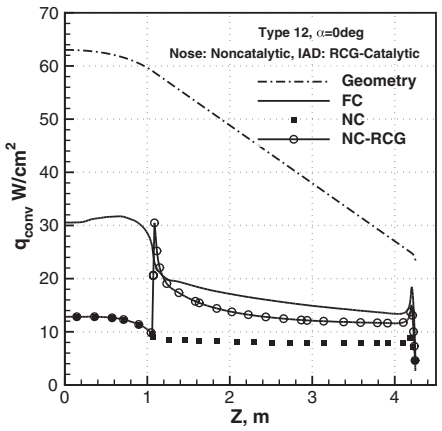

c) Surface heat flux distribution with RCG catalysis on the IAD

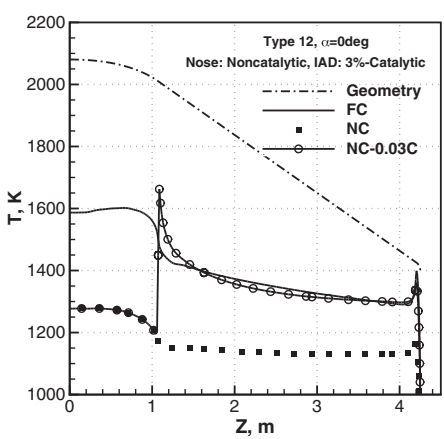

f) Surface temperature distribution with $\gamma=3 \%$ catalysis on the IAD

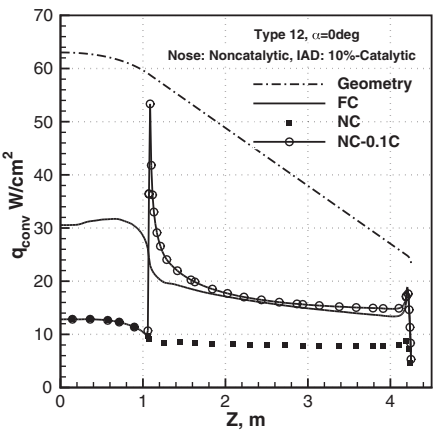

i) Surface heat flux distribution with a $10 \%$ catalytic IAD

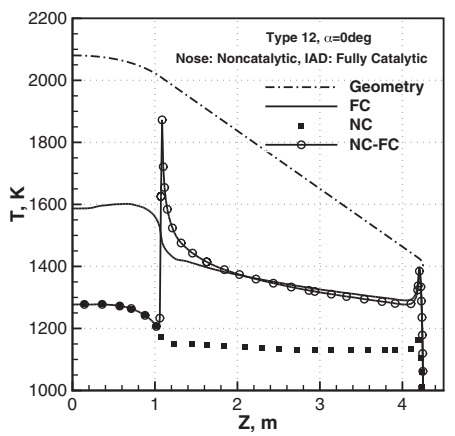

l) Surface temperature distribution with a fully-catalytic IAD

Fig. 14 Catalytic jump along the centerbody of the Type 12 geometry with a noncatalytic nose at $\alpha=0$ deg (concluded).

noncatalytic nose. For example, the MCAF for the RCG nose with a $10 \%$ catalytic IAD was only about $12 \%$ compared with $424 \%$ predicted with the noncatalytic nose. The catalytic augmentation factor for the RCG-catalytic nose was about 1.0 for IAD catalytic surfaces with $\gamma<0.10$. The catalytic jump became a catalytic undershoot with IAD catalysis less than 0.01 . Table 4 provides a summary of the RCG-catalytic nose results. A comparison of Tables 3 and 4 shows that by applying a simple RCG-catalytic coating on the nose, the catalytic augmentation factor could be reduced by a minimum of $97 \%$ as long as the IAD surface catalytic was not more than $10 \%$. This reduction reduced to about $93 \%$ with a fully catalytic IAD

To better understand the surface catalysis effect to the total heat flux, one could compute the contributions of the catalytic and conductive components of the RCG nose cases, which are tabulated in Table 5. The conduction and diffusion heat fluxes are then 
Table 3 Summary of the catalytic jump analysis for the Type 12 geometry with a noncatalytic nose at the peak heating condition and $\alpha=0 \mathrm{deg}$

\begin{tabular}{ccccc}
\hline \hline IAD catalysis, $g$ & $q_{\max }, \mathrm{W} / \mathrm{cm}^{3}$ & $T_{\max }, \mathrm{K}$ & $\mathrm{MCAF}$ & $\mathrm{MTAF}$ \\
\hline 0.00 & 12.8 & 1278 & 1.00 & 1.00 \\
0.01 & 24.3 & 1500 & 1.89 & 1.17 \\
RCG & 26.8 & 1530 & 2.08 & 1.20 \\
0.03 & 39.5 & 1690 & 3.07 & 1.32 \\
0.05 & 46.6 & 1760 & 3.62 & 1.38 \\
0.10 & 54.5 & 1830 & 4.24 & 1.43 \\
1.00 & 66.6 & 1920 & 5.19 & 1.50 \\
\hline \hline
\end{tabular}

normalized with respect to the total heat flux. These results are plotted in Fig. 16.

The results showed that the catalytic (or diffusion) heat flux increased with increasing the surface catalysis, while the conduction component remained fairly constant. The temperature gradient (conduction) influence to the total heat flux stayed a dominant factor until $\gamma \geq 0.05$, when the catalytic component surpassed the conduction component. However, for surface catalysis above $3 \%$, the total heat flux reached $100 \%$ of the corresponding fully catalytic value. That is, the total surface heat flux stayed relatively unchanged with increasing surface catalysis efficiency. Appendix B provides the

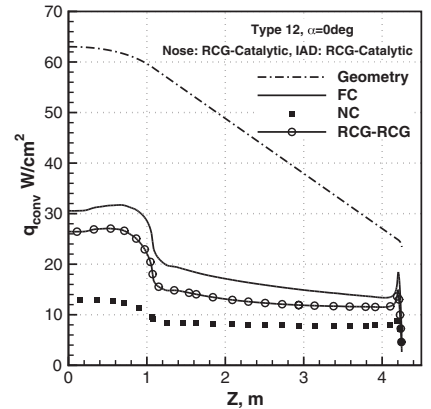

a) Surface heat flux distribution with a RCG catalytic IAD

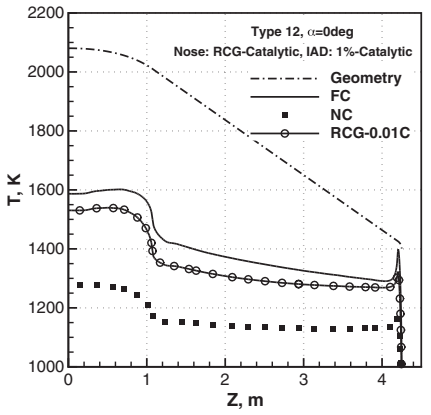

d) Surface temperature distribution with a $1 \%$ catalytic IAD

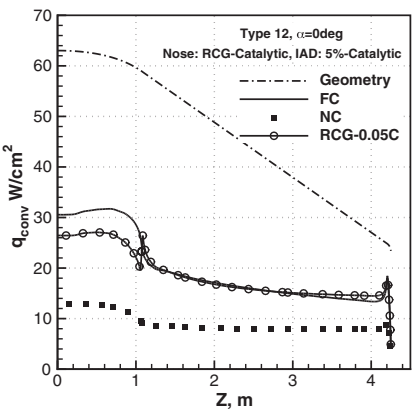

g) Surface heat flux distribution with a 5\% catalytic IAD

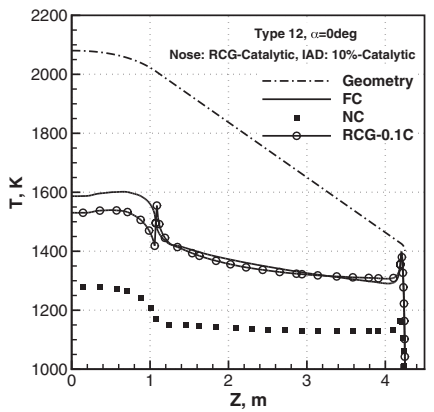

j) Surface temperature distribution with a $10 \%$ catalytic IAD

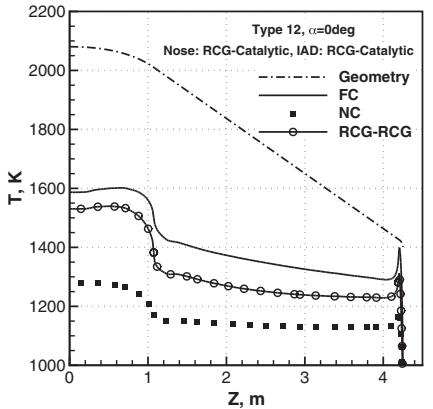

b) Surface temperature distribution with a RCG catalytic IAD

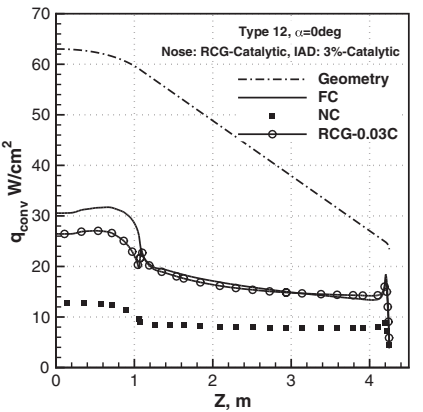

e) Surface heat flux distribution with a $3 \%$ catalytic IAD

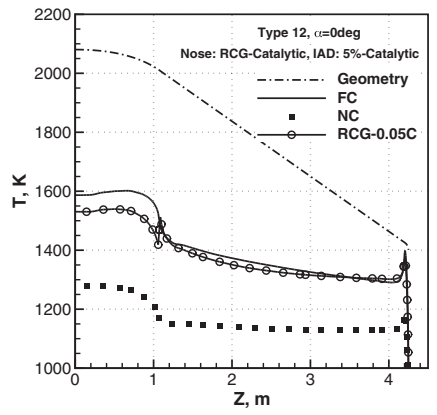

h) Surface temperature distribution with a 5\% catalytic IAD

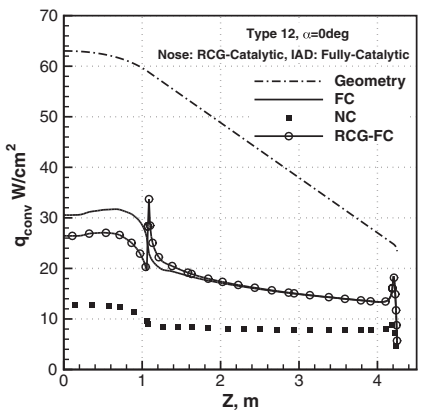

k) Surface heat flux distribution with a fully-catalytic IAD

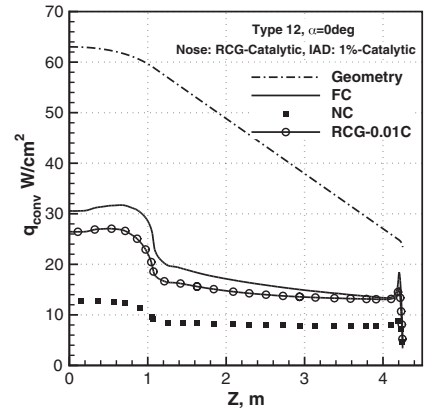

c) Surface heat flux distribution with a $1 \%$ catalytic IAD

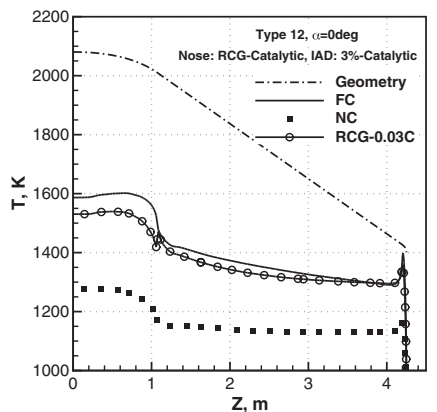

f) Surface temperature distribution with a $3 \%$ catalytic IAD

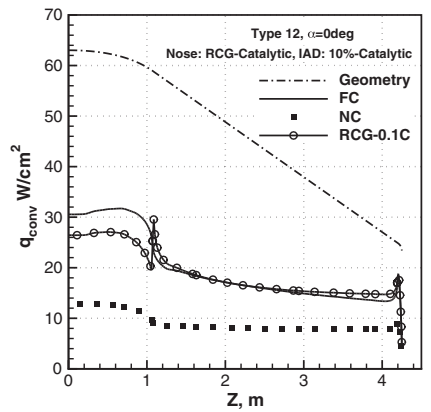

i) Surface heat flux distribution with a $10 \%$ catalytic IAD

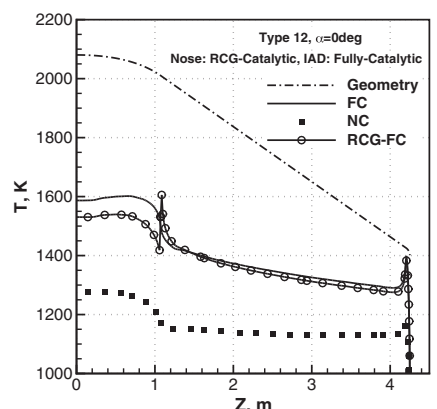

l) Surface temperature distribution with a fully-catalytic IAD

Fig. 15 Catalytic jump along the centerbody of the Type 12 geometry with a RCG-catalytic nose at $\alpha=0$ deg. 
Table 4 Summary of the catalytic jump analysis for the Type 12 geometry with a RCG-catalytic nose at the peak heating condition and $\alpha=0 \mathrm{deg}$

\begin{tabular}{ccccc}
\hline IAD catalysis, $g$ & $q_{\max }, \mathrm{w} / \mathrm{cm}^{3}$ & $T_{\max }, \mathrm{K}$ & MCAF & MTAF \\
\hline RCG & 27.2 & 1541 & 1.00 & 1.00 \\
0.01 & 27.2 & 1541 & 1.00 & 1.00 \\
0.03 & 27.2 & 1541 & 1.00 & 1.00 \\
0.05 & 27.3 & 1541 & 1.00 & 1.00 \\
0.10 & 30.5 & 1580 & 1.12 & 1.03 \\
1.00 & 34.8 & 1638 & 1.28 & 1.06 \\
\hline \hline
\end{tabular}

Table 5 Catalytic and conductive contributions to the total heat flux for different IAD surface catalysis of the Type 12 geometry with a RCG nose

\begin{tabular}{cccccc}
\hline \hline $\begin{array}{c}\text { IAD } \\
\text { catalysis, } g\end{array}$ & $\begin{array}{c}q_{\text {cond }}, \\
\mathrm{W} / \mathrm{cm}^{2}\end{array}$ & $\begin{array}{c}q_{\text {diff., }}, \\
\mathrm{W} / \mathrm{cm}^{2}\end{array}$ & $\begin{array}{c}q_{\text {total }}, \\
\mathrm{W} / \mathrm{cm}^{2}\end{array}$ & $q_{\text {cond. }} / q_{\text {total }}$ & $q_{\text {diff }} / q_{\text {total }}$ \\
\hline 0.00 & 7.9 & 0 & 7.9 & 1.00 & 0.00 \\
RCG & 8.3 & 3.5 & 11.9 & 0.70 & 0.30 \\
0.01 & 8.1 & 5.4 & 13.5 & 0.60 & 0.40 \\
0.03 & 7.8 & 6.9 & 14.8 & 0.53 & 0.47 \\
0.05 & 7.8 & 7.3 & 15.1 & 0.51 & 0.49 \\
0.10 & 7.7 & 7.6 & 15.3 & 0.50 & 0.50 \\
1.00 & 7.6 & 7.9 & 15.5 & 0.49 & 0.51 \\
\hline \hline
\end{tabular}

mathematical formulations related to the catalytic and conductive contributions to the heat flux and their physical mechanisms.

\section{Conclusions}

A series of nose-shape configurations was considered for an $8.3 \mathrm{~m}$ High-Energy Atmospheric Reentry Test (HEART) entry vehicle, and an optimum nose shape (Type 12) was recommended based on its surface heating at the peak heating point of an Earth-entry trajectory. A deployable nose mechanism was also studied during this optimization process. Angle-of-attack analysis was also done on the Type 12 geometry for which the angle-of-attack variations of \pm 10 deg were shown to have a negligible effect on the overall surface heat flux.

The surface catalysis effect on heating was also studied for a twopiece nose-inflatable aerodynamic decelerator configuration. In this configuration, the nose was not covered with the same thermal protection system fabric as it was for the case for the inflatable aerodynamic decelerator. Several nose and inflatable aerodynamic decelerator surface catalyses were considered. Accordingly, it was

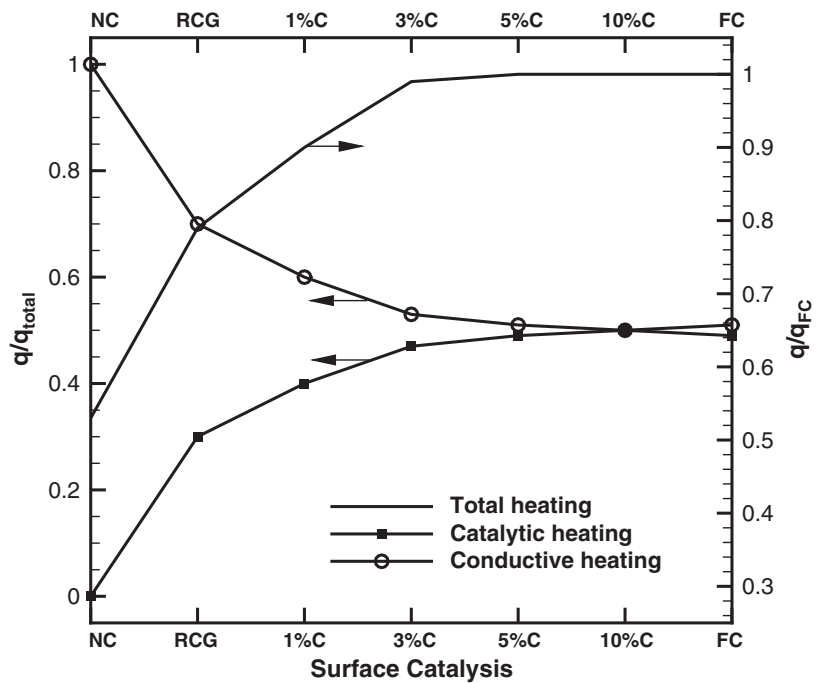

Fig. 16 Catalytic and conductive heating contributions with surface catalysis for the HEART Type 12 geometry at the peak heating point. found that thermal protection system coating with $1-2 \%$ catalytic properties, such as reaction cured glass, on the nose reduced the thermal protection system surface catalysis uncertainty on the inflatable aerodynamic decelerator by at least $93 \%$ compared with fully catalytic values. For a fabric thermal protection system with surface catalysis within $5 \%$, the minimum catalytic uncertainty reduction was about $97 \%$. Therefore, this study recommends using a catalytic coating on the rigid nose of the HEART geometry, similar to the catalytic properties studied here, to significantly reduce the catalytic uncertainty.

\section{Appendix A: Boundary-Layer Equation and Damköhler Numbers}

The results presented in Fig. 14 showed that the surface heat flux increased rapidly when the surface catalysis was increased from $\gamma=0.00$ (noncatalytic) to $\gamma=0.01$ ( $1 \%$ catalytic). However, past the nose-IAD juncture, the surface heat flux rapidly decayed approaching the fully catalytic level. For example, the surface heat flux of the IAD with $\gamma=0.01$ was about twice that of the noncatalytic surface, while the corresponding value with $\gamma \geq 0.03$ was nearly the same as the fully catalytic value. This may be explained by the gasphase recombination Damköhler number $\Gamma$ and the heterogeneous Damköhler number $\zeta$, defined as [13-17]:

$$
\Gamma=\frac{4 S c k_{r}^{\prime} T_{\mathrm{stg}}^{\omega-2}}{(\epsilon+1) \beta_{\mathrm{stg}}}\left(\frac{P_{\mathrm{stg}}}{\bar{R}}\right)^{2}
$$

$$
\zeta=\frac{S c \gamma \sqrt{\frac{\bar{R} T}{\pi M_{w}}}}{\mu_{w}} \sqrt{\frac{\rho_{w} \mu_{w}}{\beta_{\mathrm{stg}}}}
$$

where $\epsilon$ is the geometry related parameter, 0 for 2-D and 1 for axisymmetric and 3-D geometries, $\beta$ is the velocity gradient at the stagnation point, and $\omega$ is the recombination rate temperature exponent.

The gas-phase recombination Damköhler number $\Gamma$ represents the relative reaction rate's speed to the flow speed. $\Gamma$ is constant for a given body and set of reactions; the larger the body, the higher the $\Gamma$ value. $\Gamma$, however, is independent of the surface catalysis.

The heterogeneous Damköhler number $\zeta$ is representative of diffusion time to surface recombination time ration and is linearly proportional to the surface catalysis $g$; $\zeta$ is constant for a given surface catalysis and a freestream condition, but increases with a body size through $\beta$ but at a slower rate than $\Gamma$.

These nondimensional numbers were derived by writing the nonequilibirum boundary-layer equations for the species conservation and the energy in the following form $[13,16]$ :

$$
\begin{gathered}
S c f \alpha^{\prime}+\alpha^{\prime \prime}=F(\Gamma) \\
\operatorname{Pr} f \theta^{\prime}+\theta^{\prime \prime}=\frac{h_{\text {molecule }}}{C_{p} T_{e}} F(\Gamma)
\end{gathered}
$$

where $f$ is the boundary similarity variable and ()$^{\prime}=d() / d f$. The solution to these equations for surface heat flux $Q_{w}$ is [16]:

$$
Q_{w}=\underbrace{\theta^{\prime}(0)}_{\text {Continuous part }}+\underbrace{\frac{\text { Le } h_{\text {molecule }}}{C_{p} T_{e}} \alpha^{\prime}(0)}_{\text {Non-Continuous part }}
$$

This solution has two parts; a continuous part and a noncontinuous part. The second part, which is a function of the surface catalysis at the wall through $\alpha^{\prime}(0)$, is responsible for the catalytic jump presented in Figs. 14 and 15. Equation A5 is plotted versus the gas-phase recombination Damköhler number $\Gamma$ for various heterogeneous Damköhler numbers $\zeta$ in Fig. A1. In this figure, the nondimensional heat flux is defined as $Q^{*}=\left(Q-Q_{\mathrm{frzn}}\right) /\left(Q_{\mathrm{eq}}-Q_{\mathrm{frzn}}\right)$. Therefore, 


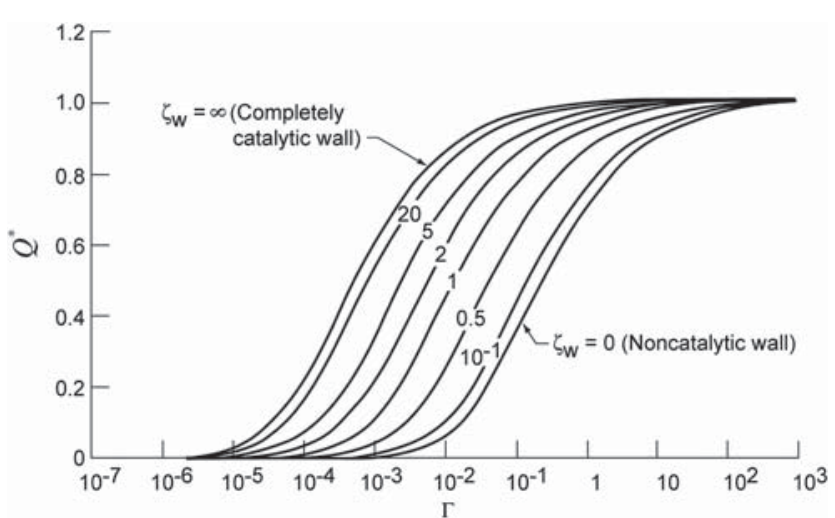

Fig. A1 Surface heat flux with Damköhler numbers; reproduced from the work of [12] (corrected labeling).

the $Q^{*}=0$ is representative of frozen heat flux, while $Q^{*}=1$ is the equilibrium solution.

This figure is used to demonstrate the surface catalysis sensitivity to the heat flux that was shown in Figs. 14 and 15. Note that the boundary-layer solution is for a very special class of problems that are somewhat different than the multibody recombinations cases conducted for the HEART at the peak heating with a radiative equilibrium boundary condition. The solution shown in Fig. A1 is for a single-body recombination with a cold surface. Thus, the solution may only be used for demonstration and better understanding of the surface catalysis effect.

For this reason, boundary-layer values and the Damköhler numbers are only extracted from the HEART computational fluid dynamic (CFD) solutions at the stagnation point for a closer representation to the boundary-layer solution. The boundary-layer solution is then used to extract the nondimensional heat flux $Q^{*}$. These are tabulated for different surface catalysis in Table A1. The results in this table show a rapid increase of heat flux as the surface catalysis increases up to $\gamma=0.03$, but this effect reaches a plateau at higher surface catalytic efficiencies. This is very similar to what is shown with the CFD analyses in Figs. 14 and 15.

\section{Appendix B: Catalytic and Conductive Heat Flux Relations with Respect to Surface Catalysis}

In Sec. V, catalytic surface effect was discussed, and the surface heat flux responses to the surface catalytic conditions were shown. Here a more detailed understanding of the surface catalytic effects to the heat flux is provided.

The heat flux equation can be separated to account for the conduction and the diffusion contributions as:

$$
q_{w}=(\underbrace{k \frac{\partial T}{\partial y}}_{\text {Conduction }}+\underbrace{\sum_{i} \rho D h_{i} \frac{\partial c_{i}}{\partial y}}_{\text {Diffusion }})
$$

where the summation is over the species. The diffusion part is indirectly a function of surface catalysis, but it is directly influenced by the species gradient at the wall. The surface catalysis changes

Table A1 Boundary-layer solution and the Damköhler numbers calculated from the HEART stagnation point at the peak heating trajectory point and $\alpha=0 \mathrm{deg}$

\begin{tabular}{cccc}
\hline \hline Catalytic efficiency, $\gamma$ & $\zeta$ & $\Gamma$ & $Q^{*}$ \\
\hline 0.01 & 0.4 & 0.5 & 0.75 \\
0.03 & 1 & 0.5 & 0.80 \\
0.05 & 2 & 0.5 & 0.85 \\
0.10 & 4 & 0.5 & 0.90 \\
1.00 & 40 & 0.5 & 1.00 \\
\hline \hline
\end{tabular}

atomic and molecular species concentration at the wall, which then influences the species gradient.

To illustrate the surface catalysis effect, consider the following reaction:

$$
N+\mathrm{N}^{\downarrow} \rightarrow N 2
$$

where $\mathrm{N}^{\downarrow}$ represents upcoming atomic nitrogen toward the wall. This is illustrated schematically in Fig. B1. For this reaction, the flux of species toward the wall must be balanced with the species diffusion at the wall:

$$
\begin{gathered}
J_{N}^{\downarrow}=J_{N} \\
\rho\left(\chi_{N}\right)_{w} \gamma \sqrt{\frac{\kappa T}{2 \pi m_{N}}}=(\rho D)_{N}\left(\frac{\partial \chi_{N}}{\partial y}\right)_{w}
\end{gathered}
$$

where $\chi$ is the species mole fraction, $\gamma$ is the surface catalytic efficiency, and $y$ is the cell height. For CFD implementation purposes, $\left(\chi_{N}\right)_{w}$ may be averaged between values in the cell above the surface and the cell below the surface (pseudocell), shown schematically in Fig. B1 as 1 and 0, respectively. The species gradient at the wall may be computed using second-order differencing. After some algebra, the atoms molar concentrations in the pseudocell are obtained as:

$$
\chi_{N}^{0}=\chi_{N}^{1} \frac{1-A}{1+A}
$$

where

$$
A=\frac{1}{2} \frac{\Delta y}{(\rho D)_{N}} \rho \gamma \sqrt{\frac{\kappa T}{2 \pi m_{N}}}
$$

For a homogeneous recombination process, the molecular molar concentration at the wall is then computed knowing that the sum of the species diffusion fluxes must be zero at the wall:

$$
\begin{gathered}
J_{N}+J_{N 2}=0 \\
(\rho D)_{N}\left(\frac{\partial \chi_{N}}{\partial y}\right)_{w}+(\rho D)_{N 2}\left(\frac{\partial \chi_{N 2}}{\partial y}\right)_{w}=0
\end{gathered}
$$

Discretizing the species gradients and rearranging Eq. B8, one may obtain the molecular concentration at the psudocells as:

$$
\chi_{N 2}^{0}=\chi_{N 2}^{1}+\left(\chi_{N}^{1}-\chi_{N}^{0}\right) \frac{(\rho D)_{N}}{(\rho D)_{N 2}}
$$

The change of species concentrations at the wall, due to the surface catalysis, indirectly affects the species rate of production throughout the boundary layer. For a thermal equilibrium condition (onetemperature), the species rate of production is presented only in the species conservation equation:

$$
\frac{\partial}{\partial t} \rho_{s}+\frac{\partial}{\partial x^{j}}\left(\rho_{s} u^{j}\right)=\frac{\partial}{\partial x^{j}}\left(\rho D_{s} \frac{\partial c_{s}}{\partial x^{j}}\right)+\dot{w}_{s}
$$

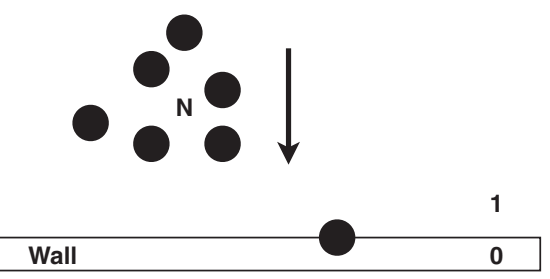

Fig. B1 Schematic of atom depositions at the wall. 
where $c_{s}$ is the species $s$ mass fraction, and $\dot{w}$ is the species mass rate of production. For generic reactions such as

$$
\alpha_{1} A+\alpha_{2} B \rightleftharpoons \beta_{1} C+\beta_{2} D
$$

where $\alpha$ and $\beta$ are Stoichiometric coefficients, the species mass rate of production is computed as

$$
\dot{w}_{s}=M_{s}\left(\sum_{s=1}^{N_{\text {react. }}}\left(\beta_{s}-\alpha_{s}\right) K_{\text {frwrd }}-\sum_{s=1}^{N_{\text {prod. }}}\left(\beta_{s}-\alpha_{s}\right) K_{\text {bkwrd }}\right)
$$

and the forward and backward reactions are computed as

$$
\begin{gathered}
K_{\text {frwrd }}=e^{a+\mathrm{blnT}-c / T} \prod_{s=1}^{N_{\text {react. }}}\left(\frac{\rho_{s}}{M_{s}}\right)^{\alpha_{s}} \\
K_{\text {bkwrd }}=e^{a+\operatorname{blnT}-c / T-\ln K_{c}} \prod_{s=1}^{N_{\text {prod. }}}\left(\frac{\rho_{s}}{M_{s}}\right)^{\beta_{s}}
\end{gathered}
$$

The equilibrium constant $K_{c}$, which is needed for computation of the backward reaction rates, is calculated from the species Gibbs free energy $G_{s}$ through the following equation:

$$
\ln K_{c}=-\left(\sum_{s=1}^{N_{\text {react. }}} \alpha_{s} G_{s}-\sum_{s=1}^{N_{\text {prod. }}} \beta_{s} G_{s}\right)-\sum_{s}\left(\alpha_{s}+\beta_{s}\right) \ln (R T)
$$

The process from Eq. B2 through Eq. B15 is repeated along with the surface temperature boundary condition until a convergence is reached. The same process can be expanded for multibody recombinations such as five-species air.

The detailed analysis of surface catalysis effect on surface heat flux provides no analytical expression for the surface heat flux, unlike the boundary-layer equation discussed in Appendix A. However, this detailed process reveals more accurate information not only at the stagnation point but also on every surface point and throughout the boundary layer. This analysis also shows the nonlinearity of the surface catalysis effect on the total heat flux as presented in Fig. 16.

\section{Acknowledgments}

The author would like to thank the hypersonic inflatable aerodynamic decelerators program and the High-Energy Atmospheric Reentry Test project for supporting this work. The thanks would also go to the entire High-Energy Atmospheric Reentry Test group for many good discussions on this effort.

\section{References}

[1] Hughes, S. J., Cheatwood, F. M., Dillman, R. A., Wright, H. S., DelCorso, J. A., and Calomino, A. M., "Hypersonic Inflatable Aerodynamic Decelerator (HIAD) Technology Development Overview," AIAA Paper 2011-2524, 2011.

[2] Reza, S., Hund, R., Kustas, F., Willcockson, W., Songer, J., and Brown, G., "Aerocapture Inflatable Decelerator (AID) for Planetary Entry," AIAA Paper 2007-2516, 2007.

[3] Hughes, S. J., Dillman, R. A., Starr, B. R., Stephan, R. A., Lindell, M. C., Player, C. J., and Cheatwood, F. M., "Inflatable Re-entry Vehicle Experiment (IRVE) Design Overview," AIAA Paper 2005-1636, 2005.

[4] Mazaheri, A. R., Gnoffo, P. A., Johnston, C. O., and Kleb, B., "LAURA 5 Users Manual," NASA TM-217092-2011, 2011.

[5] Hash, D., Olejniczak, J., Wright, M., Prabhu, D., Pulsonetti, M., Hollis, B., Gnoffo, P., Barnhardt, M., Nompelis, I., and Candler, G., "Fire II Calculations for Hypersonic Nonequilibrium Aerothermodynamics Code Verifcation: DPLR, LAURA and US3D," AIAA Paper 20070605, 2007.

[6] Mazaheri, A., and Wood, W. A., "Re-Entry Aeroheating Analysis of Tile-Repair Augers for the Shuttle Orbiter," AIAA Paper 2007-4148, 2007.

[7] Mazaheri, A., and Wood, W. A., "Heating Augmentation for Short Hypersonic Protuberances," Journal of Spacecraft and Rockets, Vol. 46, No. 2, 2009, pp. 284-291. doi: $10.2514 / 1.39992$

[8] Dyakonov, A. A., Schenenberger, M., Scallian, W. I., Van-Norman, J. W., Novak, L. A, and Tang, C. Y., "Aerodynamic Interference Due to MSL Reaction Control System,” AIAA Paper 2009-1030, 2009.

[9] Johnston, C. O., Gnoffo, P. A., and Mazaheri, A., "A Study of AblationFlowfield Coupling Relevant to the Orion Heatshield," AIAA Paper 2009-4318, 2009.

[10] Wright, M. J., White, T., and Mangini, N., "Data Parallel Line Relaxation (DPLR) Code User Manual," NASA TM-2009-215388, 2009.

[11] Scott, C. D., "Wall Boundary Equations with Slip and Catalysis for Multicomponent, Nonequilibrium Gas Flows," NASA TM-X-58111, 1973.

[12] Gupta, R. N., Scott, C. D., and Moss, J. N., "Slip-Boundary Equations for Multicomponent Nonequilibrium Airflow," NASA 2452, 1985.

[13] Fay, J. A., and Riddell, F. R., "Theory of Stagnation Point Heat Transfer in Dissociated Air," Journal of Aeronautical Sciences, Vol. 25, No. 2, 1958, pp. 373-386.

[14] Inger, G. R., "Nonequilibrium-Dissociated Boundary Layers with a Reacting Inviscid Flow," AIAA Journal, Vol. 1, No. 9, 1963, pp. 2057-2061. doi:10.2514/3.1993

[15] Inger, G. R., "Nonequilibrium Boundary-Layer Effects on the Aerodynamic Heating of Hypersonic Waverider Vehicles," Journal of Thermophysics and Heat Transfer, Vol. 9, No. 4, 1995, pp. 595-604. doi: $10.2514 / 3.713$

[16] Gnoffo, P. A., and Inger, G. R., "Analytic Corrections to CFD Heating Predictions Accounting for Changes in Surface Catalysis, Part II," AIAA Paper 96-4589, 1996.

[17] Inger, G. R., and Baker, R. L., "Nonequilibrium Viscous Shock-Layer Heat Transfer with Arbitrary Surface Catalycity," Journal of Spacecraft and Rockets, Vol. 42, No. 2, 2005, pp. 193-200. doi: $10.2514 / 1.7568$

G. Palmer Associate Editor 